\title{
Tomada de decisão de estudantes frente a um case scenario de Endodontia
}

\author{
Gianna Espínola Moura*; José Régis Cordeiro da Silva**; Lorena Marques da Nóbrega***; Katia \\ Simone Alves dos Santos****; Sérgio d'Avila****
}

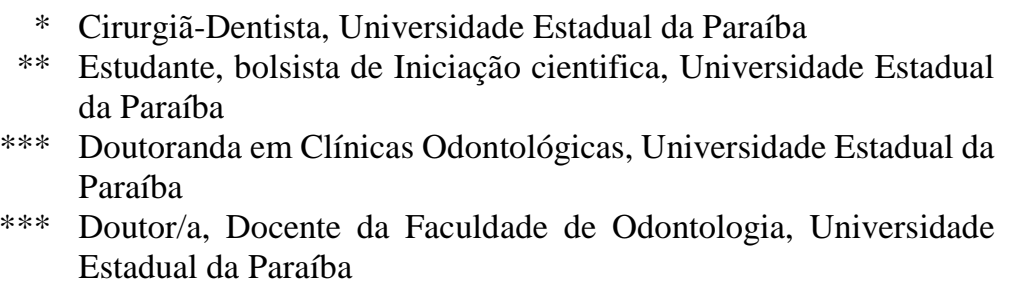

Recebido em 16/05/2017. Aprovado em 22/10/2017.

\begin{abstract}
RESUMO
O presente estudo do tipo transversal avalia o processo de tomada de decisão de estudantes do $4^{\circ}$ e $5^{\circ}$ anos do curso de Odontologia de uma universidade pública da região Nordeste, frente a um case scenario de Endodontia. A amostra foi constituída por 80 estudantes, os quais, frente ao cenário descrito e imagem radiográfica apresentada, responderam a um questionário sobre o provável motivo da rarefação, a conduta clínica e a tomada de decisão. Para análise dos dados, foram obtidas distribuições absolutas, percentuais uni e bivariadas. A maioria dos pesquisados foi do sexo feminino $(55,0 \%)$. Analisando a provável causa da rarefação periapical, 43,8\% dos entrevistados acreditam que se deva ao limite apical de obturação. A anamnese, o exame clínico e a radiografia foram mencionados como os meios semiotécnicos indicados para a tomada de decisão de tratamento no caso apresentado por $95 \%$ dos estudantes. A maioria $(60 \%)$ decidiu pelo retratamento endodôntico utilizando medicação intracanal. Conclui-se que os estudantes consideram o limite apical de obturação indesejável como motivo da rarefação apical; que a anamnese, exame clínico, exame radiográfico são indicados para a tomada de decisão e optaram pelo retratamento endodôntico. Os estudantes do $5^{\circ}$ ano, com mais vivência clínica que os do $4^{\circ}$, apresentaram decisão terapêutica mais conservadora.
\end{abstract}

Descritores: Ensino Superior. Tomada de Decisão Clínica. Endodontia. Falha de Tratamento.

\section{INTRODUÇÃO}

As Diretrizes Curriculares Nacionais (DCN $)^{1}$ para os cursos de Odontologia objetivam promover a formação de competências para que os discentes possam resolver os problemas de saúde bucal da população. $O$ conjunto de mudanças curriculares incentiva a perspectiva de aprendizado pela vivência, pela sensibilidade, 
pela compreensão da realidade social e pelo olhar humano, sem perder a excelência técnica e os fundamentos teóricos-científico ${ }^{2}$. Assim, o professor como facilitador e mediador do ensinoaprendizagem deve incentivar a tomada de decisão pelo os alunos, como ferramenta para consolidar o aprendizado.

O processo de tomada de decisão não pode ser considerado uma tarefa fácil e pode criar muitas situações de posições contraditórias e o conflito é inevitável. As questões éticas envolvidas podem tornar o processo mais complexo, mas fornecem, sem dúvida, uma referência importante ${ }^{3}$.

$\mathrm{Na}$ Odontologia, pode haver também dilemas entre o ponto de vista da norma e o ponto de vista descritivo ao tomar uma decisão. A tarefa do cirurgião-dentista ao escolher o tratamento ideal para cada paciente requer uma avaliação racional dos riscos envolvidos. Há evidências de que as características do paciente, do profissional e do trabalho influenciam especificamente as decisões tomadas pelos cirurgiões-dentistas quanto aos tratamentos ${ }^{3,4}$.

As alterações pulpares e/ou periapicais podem dar origem a várias patologias que frequentemente determinam a necessidade de intervenção endodôntica ${ }^{5}$. O estabelecimento de um padrão para o controle de qualidade do tratamento endodôntico é importante ao se determinar o sucesso endodôntico, sendo um fator relevante a ser considerado à seleção de casos. O diagnóstico clínico do insucesso do tratamento endodôntico, realizado pela anamnese, exames clínico e radiográfico, determina condições que indicam as opções de tratamento ${ }^{6,7}$. Muitas vezes, o diagnóstico das lesões pulpares e periapicais apresenta características tão semelhantes que exige atenção especial na observância dos detalhes diferenciais ${ }^{8}$.

Em alguns casos pode ser relativamente simples tomar a decisão em um tratamento; em muitos outros, torna-se mais complexo. Nem todas as decisões de intervir trazem benefícios, e nem todas as decisões de não intervir são prejudiciais. Tratamentos podem produzir efeitos indesejáveis e programar alguns procedimentos de diagnóstico pode ser arriscado ou mesmo desnecessário ${ }^{6}$.

Para se concluir que o tratamento instituído teve êxito, é necessário que na proservação se observe a ausência de dor, provocada ou espontânea; edema; desaparecimento de fístula preexistente; sensível diminuição ou total remissão de rarefações ósseas periapicais e presença da lâmina dura. Todavia, se durante a proservação realizada num período de seis meses a dois anos, detecta-se presença de dor, edema, mobilidade, fístula, sensibilidade à percussão, o não desaparecimento ou diminuição e ainda, o eventual aumento de lesão periapical preexistente, assim, como o surgimento de rarefação óssea periapical não detectada anteriormente, se está diante de um fracasso endodôntico, devendo-se identificar as causas do insucesso, de modo a programar o retratamento de maneira adequada ${ }^{6,9}$.

Neste contexto, o presente estudo teve por objetivo avaliar, a partir da de um cenário de rarefação apical em um dente endodonticamente tratado, a resposta dos estudantes quanto ao motivo da rarefação e à decisão de conduta clínica.

\section{METODOLOGIA}

A tipologia clássica deste estudo transversal teve uma característica particular, pois se utilizou um "cenário de casos" (case scenarios). Estes já são conhecidos em pesquisas epidemiológicas e têm sido utilizados na maioria dos estudos específicos de tomada de decisão ${ }^{10}$. Utilizados como método por d'Avila $(2006)^{3}$ é considerado um método de grande valor, apresentando a 
vantagem distinta de controlar variáveis, os dados foram coletados por meio de permitido analisar a extensão pela qual elas são questionário (figura 2).

responsáveis por diferenças em tomada de decisão.

Foi realizado com estudantes do curso de Odontologia da Universidade Estadual da Paraíba, localizada na Cidade de Campina Grande/PB. Este município é considerado um dos principais polos de desenvolvimento científico e tecnológico da região Nordeste do Brasil. Segundo dados da coordenação do curso, na época da coleta dos dados havia 290 alunos matriculados. Os 99 estudantes matriculados nos quatro últimos períodos e que já haviam cursado a disciplina de Endodontia foram incluídos na amostra.

O estudo está em conformidade com as normas internacionais (Declaração de Helsinque) e a legislação nacional (Resolução 466/12 do Conselho Nacional de Saúde) que regem a ética em estudos envolvendo seres humanos e foi autorizado pela instituição em que foi conduzido, tendo sido avaliado e aprovado por um comitê de ética em Pesquisa independente (CAAE 0152.0.133.000-09). O termo de consentimento livre e esclarecido para os pesquisados foi formulado e os que se recusaram a participar do estudo foram computados como não respondentes.

Os estudantes receberam a seguinte descrição: paciente do sexo feminino, 37 anos, em tratamento odontológico nas clinicas de uma universidade pública, relatou que havia sido submetida a um tratamento endodôntico no dente 22 há um ano. Ao exame, não apresenta dor, edema ou fístula na região. Ausência de sinais positivos à percussão, tanto vertical como horizontal. Na radiografia periapical (figura 1) observa-se a imagem de um dente endodonticamente tratado, com obturação e selamento coronário aparentemente satisfatórios, apresentando rarefação óssea periapical. A seguir,

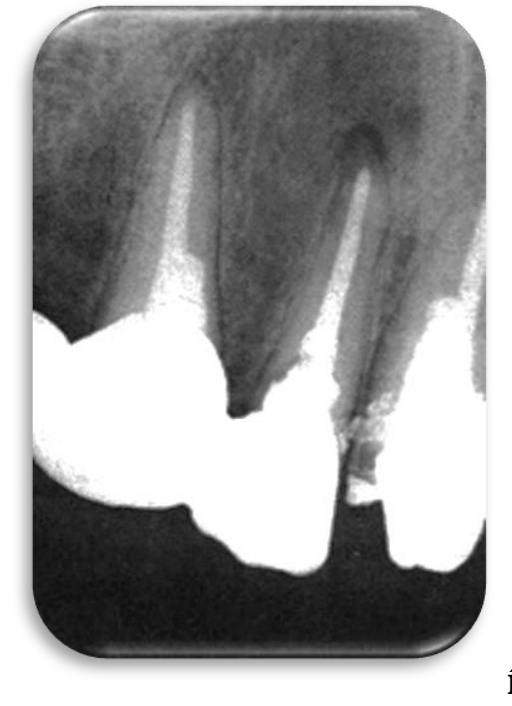

Figura 1. Radiografia apresentada

Dados do estudante:

Sexo____ Idade___ Ano que cursa_

1. De acordo com a descrição do caso quais os fatores que levaram à radiolucidez apical, mesmo após o tratamento endodôntico?

A. Somente a não utilização de medicação intracanal durante o tratamento

B. Somente o selante coronário insatisfatório durante ou após o tratamento

C. Somente o limite apical de obturação indesejável

D. Todas as opções

E. Nenhuma opção

F. Opções A e C

2. Qual a sua conduta para elucidar o caso?

A. Anamnese, exame clínico, exame radiográfico

B. Exame clínico, exame radiográfico

3. Quais as possíveis alternativas de tratamento?
A. Proservação por seis meses a um ano
B. Retratamento em uma única sessão
C. Retratamento utilizando de medicação intracanal
D. Retratamento seguido de curetagem periapical
E. Complementação cirúrgica

Figura 2. Questionário para coleta de dados 
Os dados foram digitados em planilha Excel, e o software estatístico utilizado para a obtenção dos cálculos foi o Statistical Analysis System - SAS. O nível de significância utilizado nas decisões dos testes estatísticos foi de 5,0\%.

\section{RESULTADOS}

Dos 99 estudantes matriculados nos dois últimos anos do curso, 80 se dispuseram a participar do estudo, correspondendo a $80,8 \%$ da amostra. A idade variou de 20 a 42 anos. $\mathrm{Na}$ tabela 1 é possível destacar que a maioria da amostra foi composta de pesquisados do sexo feminino $(55,0 \%)$, a faixa etária mais prevalente foi a de 20 a 22 anos, representando $37,5 \%$ dos pesquisados e a amostra foi homogeneamente distribuída entre estudantes do $4^{\circ}$ e $5^{\circ}$ ano.

Nas respostas apresentadas, $43,8 \%$ dos pesquisados consideraram que somente o limite apical de obturação insatisfatória seria a causa provável da radiolucidez. Analisando a variável tomada de decisão, 95\% dos entrevistados consideram que a anamnese, seguida de exame clínico e radiográfico seria a decisão mais sensata para estabelecer o diagnóstico e a conduta a ser tomada. Quanto à variável conduta, indicariam o retratamento endodôntico utilizando medicação intracanal (60\%) (tabela 2).

Em relação à tomada de decisão e o ano de curso não se verificam diferenças percentuais elevadas (tabela 3), visto que a maior diferença foi de $5 \%$ no exame clínico entre os alunos do $4^{\circ}$ e $5^{\circ}$ ano do curso.

$\mathrm{Na}$ tabela 4 verifica-se que o percentual de estudantes que escolheriam o retratamento utilizando a medicação intracanal foi significativamente mais elevado entre os do $4^{\circ}$ ano em relação aos do $5^{\circ}$ ano (teste de ratio, $\mathrm{p}<0,05$ ).

Tabela 1. Distribuição dos entrevistados segundo as variáveis: sexo, idade e ano que cursa

\begin{tabular}{lll}
\hline Variável & $\mathbf{n}$ & \% \\
\hline Sexo & 36 & \\
Masculino & 44 & 55,0 \\
Feminino & & \\
Idade & 30 & 37,5 \\
20 a 22 & 27 & 33,8 \\
23 a 24 & 23 & 28,8 \\
25 a mais & & \\
Ano que cursa & 40 & 50,0 \\
$4^{\circ}$ ano & 40 & 50,0 \\
$5^{\circ}$ ano & $\mathbf{8 0}$ & $\mathbf{1 0 0}$ \\
TOTAL & & \\
\hline
\end{tabular}


Tabela 2. Distribuição dos entrevistados segundo as variáveis motivo da radiolucidez, tomada de decisão e conduta

\begin{tabular}{lcc}
\hline Variável & $\mathbf{n}$ & $\mathbf{\%}$ \\
\hline Radiolucidez & & \\
A. Somente a não utilização de medicação intracanal durante o tratamento & 06 & 7,5 \\
B. Somente o selante coronário insatisfatório durante ou após o tratamento & 02 & 2,5 \\
C. Somente o limite apical de obturação indesejável & 35 & 43,8 \\
D. Todas as opções & 27 & 33,8 \\
E. Nenhuma opção & 06 & 7,5 \\
F. Opções A e C & 04 & 4,9 \\
Tomada de decisão & & \\
A. Anamnese, exame clínico, exame radiográfico & 76 & 95,0 \\
B. Exame clínico, exame radiográfico & 04 & 5,0 \\
Conduta & & \\
A. Proservação por um período de seis meses a um ano & 18 & 22,5 \\
B. Retratamento em uma única sessão & 01 & 1,3 \\
C. Retratamento utilizando de medicação intracanal & 48 & 60,0 \\
D. Retratamento seguido de curetagem periapical & 05 & 6,3 \\
E. Complementação cirúrgica & 08 & 10,0 \\
TOTAL & $\mathbf{8 0}$ & $\mathbf{1 0 0}$ \\
\hline
\end{tabular}

Tabela 3. Distribuição dos pesquisados segundo as variáveis tomada de decisão e ano do curso

\begin{tabular}{lcccccc}
\hline \multirow{2}{*}{ Decisão } & \multicolumn{4}{c}{ Ano do curso } \\
& \multicolumn{2}{c}{$\mathbf{4}^{\mathbf{0}}$ ano } & \multicolumn{2}{c}{$\mathbf{5}^{\mathbf{0}}$ ano } & \multicolumn{2}{c}{ Total } \\
& $\mathbf{n}$ & $\mathbf{\%}$ & $\mathbf{n}$ & $\mathbf{\%}$ & $\mathbf{n}$ & $\mathbf{\%}$ \\
\hline A. Anamnese, exame clínico, exame radiográfico & 37 & 46,3 & 39 & 48,8 & 76 & 95,0 \\
B. Exame clínico, exame radiográfico & 03 & 7,5 & 01 & 2,5 & 04 & 5,0 \\
TOTAL & 40 & 50,0 & 40 & 50,0 & 80 & 100,0 \\
\hline
\end{tabular}

Teste de ratio

Tabela 4. Distribuição dos pesquisados segundo as variáveis: conduta e ano do curso

\begin{tabular}{lcccccc}
\hline \multirow{2}{*}{ Conduta } & \multicolumn{3}{c}{ Ano de Curso } & \multicolumn{2}{c}{ Total } \\
& $\mathbf{4}^{\mathbf{0}}$ ano & \multicolumn{2}{c}{$\mathbf{5}^{\mathbf{0}}$ ano } & \multicolumn{2}{c}{ Tota } & \% \\
\hline A. Proservação por um período de seis meses a um ano & 04 & 5,0 & 14 & 8,8 & 18 & 22,5 \\
B. Retratamento em uma única sessão & 01 & 1,3 & - & - & 01 & 1,3 \\
C. Retratamento utilizando de medicação intracanal & 29 & 36,3 & 19 & 23,8 & 48 & 60,0 \\
D. Retratamento seguido de curetagem periapical & 02 & 2,5 & 03 & 3,8 & 05 & 6,3 \\
E. Complementação cirúrgica & 04 & 5,0 & 04 & 5,0 & 08 & 10,0 \\
TOTAL & 40 & 50,0 & 40 & 50,0 & 80 & 100,0 \\
\hline Teste de ratio & & & & & &
\end{tabular}

Teste de ratio 


\section{DISCUSSÃO}

Um dos grandes desafios para o atendimento clínico em Odontologia está na decisão sobre o procedimento a ser realizado. A tomada de decisão geralmente é técnica, mas também intuitiva. Portanto, requer um olhar investigativo dos procedimentos que devem interagir com a compreensão da individualidade do paciente, o que inviabiliza e exclui qualquer abordagem de caráter universal.

A vivência da tomada de decisão pelo estudante contribui para o melhor entendimento do conteúdo, com a integração e aplicação do conhecimento para os cuidados específicos centrados no paciente, orienta quanto às atitudes corretas a se tomar e favorece o desenvolvimento de competências por meio da prática.

Os estudantes demonstraram interesse em participar do estudo (taxa de resposta de 80,8\%). Dessa forma, enfatiza o uso da ferramenta case scenarios para consolidar o ensino de Odontologia e despertar o rigor técnico científico do aluno, por meio do olhar investigativo frente a uma tomada de decisão.

Os dados obtidos neste estudo mostram que a maioria dos entrevistados $(33,8 \%)$ afirmou que vários fatores poderiam influenciar na manutenção de uma radiolucidez periapical, como a não utilização de medicação intracanal; selamento coronário insatisfatório durante ou após obturação e ainda, limite apical de obturação insatisfatório. Apenas 2,5\% dos entrevistados afirmaram que o não adequado selamento coronário durante o tratamento pode ser a causa da ocorrência de radiolucidez periapical após o tratamento endodôntico.

Em casos de necrose pulpar, especialmente na presença de lesões perirradiculares, a obturação do canal deve ser realizada em uma segunda sessão, após a permanência de medicação intracanal por um período variável de três a sete dias ${ }^{11-19}$.
Um canal pode ser recontaminado e reinfectado entre as sessões de tratamento por meio de microinfiltração através do material selador temporário, por sua degradação ou fratura, podendo acarretar o insucesso da terapia endodôntica ${ }^{20}$. Neste sentido, estudos concluíram que a resina composta e o ionômero de vidro se mostraram efetivos para minimizar a micro infiltração coronária ${ }^{21-24}$.

Quando questionados sobre o que consideram importante para a tomada de decisão de tratamento, 95\% dos alunos entrevistados apontaram a anamnese, exame clínico e exame radiográfico. Não apenas o exame radiográfico é importante, até porque a literatura citada demonstra que reparo de lesões periapicais depende também de fatores sistêmicos do paciente e esta informação só é conseguida mediante anamnese e exame clínico ${ }^{25}$.

Cerca de 1/4 dos alunos entrevistados $(22,5 \%)$ afirmaram que optariam pela proservação por seis meses a um ano. Observouse que os estudantes do $5^{\circ}$ ano $(8,8 \%)$ demonstram maior noção da necessidade de acompanhamento do que os do $4^{\circ}$ ano (5\%), que parecem ser mais imediatistas na resolução dos problemas. Pode-se justificar este fato à maior vivência. Evidencia-se, assim, a necessidade de rediscutir a ideia de proservação de um caso com poucas implicações do insucesso, principalmente quando não se tem informações sobre o tratamento prévio realizado.

Em um estudo utilizando a tomada de decisão sobre a substituição de restaurações, os pesquisados tiveram que optar, através de imagens de restaurações, por uma entre três opções terapêuticas: trocar; reparar ou manter a restauração. Houve tendência à adoção de terapias mais conservadoras, mantendo e reparando as restaurações em vez de substituílas, havendo uma necessidade de uma discussão continua sobre critérios mais conservadores de 
tomadas terapêuticas e não intervencionistas ${ }^{26}$.

Em casos de dentes com lesão perirradicular, o sucesso do tratamento depende do nível de obturação em relação ao ápice radicular. Assim, canais obturados no limite do ápice ou até 2 milímetros aquém apresentaram 94\% de sucesso, enquanto os sobreobturados ou os obturados a mais de 2 milímetros do ápice apresentaram índices menores, ou seja, $76 \%$ e $68 \%$, respectivamente ${ }^{9,27}$.

Alguns aspectos devem ser considerados para a adequada tomada de decisão frente às situações clínicas que envolvam rarefação periapical. Não existe relação entre o limite no qual o canal é obturado e o sucesso ${ }^{9}$. Não se deve imaginar que obturar a 1 ou 2 milímetros seja o fator determinante para se atingir o sucesso, pois não é a extensão desse espaço que determina isso e sim as condições em que ele se encontra. $\mathrm{Na}$ polpa necrosada, apesar do rigor do limite apical da obturação, sem perceber, os autores relatam a ocorrência de reparo diante das mais diversas medidas $^{28}$.

Optando-se por um retratamento endodôntico, a maioria dos alunos (60\%) acredita que este deve ser realizado em mais de uma sessão com uso de medicação intracanal entre as mesmas. A interpretação deste dado confronta com os resultados do questionamento sobre, se a manutenção da radiolucidez periapical seria decorrente do não uso de uma medicação intracanal, onde apenas 7,5\% dos entrevistados afirmaram saber da importância da medicação para prevenção de patologias periapicais.

A valorização da tomada de decisão é fruto da política de DCN em Odontologia, que incentiva a adoção de novas estratégias no processo de ensino ${ }^{4,29}$. Essas novas práticas se consolidam melhor quando é promovida a aproximação do estudante com a realidade de trabalho, e as competências se configuram como premissas fundamentais para resolução eficiente dos problemas.

\section{CONCLUSÃO}

A maioria dos estudantes considerou somente o limite apical de obturação indesejável como motivo da rarefação apical; que a anamnese, exame clínico, exame radiográfico são indicados e optou pelo retratamento endodôntico. Os estudantes do $5^{\circ}$ ano, com mais vivência clínica que os do $4^{\circ}$, apresentaram decisão terapêutica mais conservadora.

\section{ABSTRACT \\ Students' decision-making in the face of an endodontic case scenario}

This cross-sectional study evaluates the decisionmaking process of students in the 4 th and 5 th years of the Dentistry course of a public university in the Northeast region, facing a case scenario of Endodontics. The sample consisted of 80 students, who, in view of the described scenario and radiographic image presented, answered a questionnaire about the probable reason for rarefaction, clinical conduct and decision making. For data analysis, absolute distributions, uni, and bivariate percentages were obtained. The majority of respondents were female $(55.0 \%)$. Analyzing the probable cause of periapical rarefaction, $43.8 \%$ of respondents believe that it is due to the apical limit of obturation. Anamnesis, clinical examination, and radiography were mentioned by $95 \%$ of the students as the means indicated for the treatment decision-making in the case presented. The majority $(60 \%)$ decided for endodontic retreatment using intracanal medication. It is concluded that the students consider the undesirable apical limit of obturation as a reason for apical rarefaction; that anamnesis, clinical examination, radiographic examination are indicated for decision making and have chosen endodontic retreatment. The students of the 5th year, with more clinical experience than those of the 4th, presented a more conservative therapeutic decision. 
Descriptors: Education, Higher. Clinical Decision-Making. Endodontics. Treatment Failure.

\section{REFERÊNCIAS}

1. Brasil. Ministério da Educação. Parecer CNE/CES no 1.300/2001. Diário Oficial da União. Brasília; 2001; p. 25

2. Gabriel M, Tanaka EE. Formação profissional em Odontologia: percepção discente da interação curricular. Rev ABENO. 2011; 11(1):19-22.

3. d'Ávila S, Cavalcanti AL, Caldas Jr AF. Selection of patients by dentists in a situation where health resources are scarce. Acta Bioethica. 2012;18(2):173-80.

4. Murgic L, Hébert PC, Sovic S, Pavlekovic G. Paternalism and autonomy: views of patients and providers in a transitional (postcommunist) country. BMC Med Ethics. 2015; 16(1):65.

5. Larsen $\mathrm{T}$, Fiehn NE. Dental biofilm infections - an update.

APMIS. 2017; 125(4):376-84

6. Çiçek E, Özsezer-Demiryürek E, ÖzerolKeskin NB, Murat N. Comparison of treatment choices among endodontists, postgraduate students, undergraduate students and general dentists for endodontically treated teeth. Int Dent J. 2016 ;66(4):201-7

7. Estrela C, Holland R, Estrela CR, Alencar AH, Sousa-Neto MD, Pécora JD. Characterization of successful root canal treatment. Braz Dent J. 2014; 25(1):3-11.

8. Abu-Tahun I, Rabah'ah A, Khraisat A. A review of the questions and needs in endodontic diagnosis. Odontostomatol Trop. 2012;35(140):11-20.

9. Lopes HP, Siqueira Jr JF. Endodontia biologia e técnica. Rio de Janeiro: Elsevier; 2015. 848 p.
10. Mackinlan JB, Potter DA, Feldman HA. Non-medical influences on medical decision-making. Soc Sci Med. 1996;42(5):769-76.

11. Consolaro A. Dental concussion: suggested protocol for controlling its consequences, treatment, prognosis and follow-up. Dental Press Endod. 2015;5(3):10-20.

12. Nair PN, Henry S, Cano V, Vera J. Microbial status of apical root canal system of human mandibular first molars with primary apical periodontitis after "one-visit" endodontic treatment. Oral Surg Oral Med Oral Pathol Oral Radiol Endod. 2005; 99(2):231-52.

13. Mohammadi Z, Shalavi S, Yazdizadeh M. Antimicrobial activity of calcium hydroxide in endodontics: a review. Chonnam Med J. 2012;48(3):133-40.

14. Sokhi RR, Sumanthini MV, Shenoy VU, Bodhwani MA. Effect of calcium hydroxide based intracanal medicaments on the apical sealing ability of resin based sealer and guttapercha obturated root canals. J Clin Diagn Res. 2017;11(1):75-9.

15. Siqueira Jr JF, Lopes HP. Medicação intracanal. In: Lopes HP, Siqueira JF Jr, Endodontia biologia e técnica, 2ed. Ed. Rio de Janeiro: Guanabara Koogan; 2004. p. 581-618.

16. Anjaneyulu K, Nivedhitha MS. Influence of calcium hydroxide on the post-treatment pain in Endodontics: A systematic review. J Conserv Dent. 2014;17(3):200-7.

17. Mohammadi Z, Dummer PM. Properties and applications of calcium hydroxide in endodontics and dental traumatology. Int Endod J. 2011;44(8):697-730

18. Smaïl-Faugeron V, Courson F, Durieux P, Muller-Bolla M, Glenny AM, Fron Chabouis H. Pulp treatment for extensive 
decay in primary teeth. Cochrane Database Syst Rev. 2014. (8):CD003220

19. Olatosi OO, Sote EO, Orenuga OO. Effect of mineral trioxide aggregate and formocresol pulpotomy on vital primary teeth: a clinical and radiographic study. Niger J Clin Pract. 2015;18(2):292-6.

20. Srivastava PK, Nagpal A, Setya G, Kumar S, Chaudhary A, Dhanker K . Assessment of coronal leakage of temporary restorations in root canal-treated teeth: an in vitro study. J Contemp Dent Pract. 2017;18(2):126-30.

21. Zarenejad N, Asgary S, Ramazani N, Haghshenas MR, Rafiei A, Ramazani M. Coronal microleakage of three different dental biomaterials as intra-orifice barrier during nonvital bleaching. Dent Res J. 2015;12(6):581-8.

22. Ceyhanli KT, Orucoglu H, Erdilek N, Turkun M, Akdağ MS. Microleakage of 4 post-luting cements evaluated by the computerized fluid filtration method. Int $\mathrm{J}$ Artif Organs. 2015;38(12):646-50.

23. Matos NHR, Pimenta AC Jr, Melo LL. Análise da infiltração em materiais em três tipos de restauradores provisórios de uso em endodontia. J Bras Endod. 2003; 4(2): 1538.

24. Savadkouhi ST, Bakhtiar H, Ardestani SE. In vitro and ex vivo microbial leakage assessment in endodontics: A literature review. J Int Soc Prev Community Dent. 2016; 6(6):509-16.

25. Givol N, Rosen E, Taicher S, Tsesis I. Risk management in endodontics. J Endod. 2010; 36(6):982-4.
26. Santos PHB, Alencar AV Filho, Pedrosa Guimarães R, Vicente Silva CH. Manter, reparar ou substituir restaurações? Tomada de decisão terapêutica dos estudantes do curso de Odontologia da UFPE. Int J Dent. 2008; 7(3):147-52.

27. Sjogren U, Hagglund B, Sundqvist G, Wing $\mathrm{K}$. Factors affecting the long-term results of endodontic treatment. J Endod. 1990; 16(10): 498-504.

28. Pamboo J, Hans MK, Chander S, Sharma K. Endodontic Management of Maxillary First Molar With Two Palatal Canals Aided With Cone Beam Computed Tomography: A Case Report. Compend Contin Educ Dent. 2017;38(4):1-4.

29. Hasini B, Vishna DN, Srinivasan R, Afshan S, Sneha R, Kamalan J, et al. Student preparedness characteristics important for clinical learning: perspectives of supervisors from medicine, pharmacy and nursing. BMC Med Educ. 2017; 17(130): 1-9.

Correspondência para:

Sérgio d'Avila

e-mail: davila2407@hotmail.com

Departamento de Odontologia

Universidade Estadual da Paraíba

Av. das Baraúnas, 351, Bairro Universitário 58429-500, Campina Grande/PB 